\title{
B-Elemene inhibits proliferation of human glioblastoma cells and causes cell-cycle G0/G1 arrest via mutually compensatory activation of MKK3 and MKK6
}

\author{
TINGZHUN ZHU ${ }^{1}$, YONGSHUN ZHAO ${ }^{1}$, JIANING ZHANG ${ }^{2}$, LONGJIE LI ${ }^{3}$, \\ LIJUAN ZOU ${ }^{3}$, YIQUN YAO ${ }^{1}$ and YINGHUI XU ${ }^{1}$ \\ ${ }^{1}$ Department of Neurosurgery, The First Affiliated Hospital of Dalian Medical University, No. 222 Zhong Shan Road, \\ Dalian 116011; ${ }^{2}$ Department of Biochemistry, Dalian Medical University, No. 9 West Segment of South \\ Lvshun Road, Dalian 116044; ${ }^{3}$ Department of Radiation Oncology, The Second Affiliated Hospital \\ of Dalian Medical University, No. 467 Zhong Shan Road, Dalian 116023, P.R. China
}

Received July 21, 2010; Accepted September 21, 2010

DOI: $10.3892 /$ ijo.2010.855

\begin{abstract}
Elemene, a natural plant drug extracted from Curcuma wenyujin, has shown a strong anti-glioblastoma effect. However, the antitumor mechanism of $\beta$-elemene remains unclear. Mitogen-activated protein kinase kinase-3 (MKK3) and -6 (MKK6) can regulate cellular growth, fission, differentiation and apoptosis. To illustrate the role of MKK3 and MKK6 in the anti-glioblastoma proliferation effect of ß-elemene, U87 cells were treated with $\beta$-elemene at various doses or for different times, and then phosphorylated MKK3 (p-MKK3), phosphorylated MKK6 (p-MKK6), MKK3 and MKK6 were detected by Western blot assay. After transient transfection with dominant-negative mutant plasmids of MKK3 and MKK6, cell viability and cell cycle stage were determined by methyl thiazolyl tetrazolium assay and flow cytometry, respectively. Results showed that B-elemene inhibited the proliferation of U87 glioblastoma cells and arrested them in $\mathrm{G} 0 / \mathrm{G} 1$ phase through up-regulating p-MKK3 and p-MKK6 levels. In contrast, inhibition of MKK3 and MKK6 reversed the antitumor effect of $B$-elemene. Furthermore, when either MKK3 or MKK6 was inhibited by a dominantnegative plasmid, the other was compensatorily activated in the presence of $\beta$-elemene. Taken together, our findings indicate that mutually compensatory activation of MKK3 and MKK6 mediates the anti-glioblastoma effect of B-elemene. MKK3 and MKK6 might be two putative targets for molecular therapy against glioblastoma.
\end{abstract}

Correspondence to: Dr Yinghui Xu, Department of Neurosurgery, The First Affiliated Hospital of Dalian Medical University, No. 222 Zhong Shan Road, Dalian 116011, P.R. China

E-mail: xuyh@dmu-1.com

Key words: ß-Elemene, glioblastoma, MKK3, MKK6

\section{Introduction}

Glioblastoma is the most common and aggressive type of primary brain tumor, accounting for approximately $52 \%$ of all primary intracranial tumors and $20 \%$ of all brain neoplasms. Despite recent advances in the therapeutics of surgical resection, chemotherapy and radiotherapy, it is still difficult to efficiently treat glioblastoma, and the prognosis for patients with glioblastoma remains dismal (1-4).

Elemene, an effective antitumor medicine, is extracted from natural plant Curcuma wenyujin and exists as an essential oil mixture of $\beta-, \gamma$ - and $\delta$-elemene (5). As the major active antitumor component, $\beta$-elemene has been isolated as a monomeric drug and found to strongly induce apoptosis and antiproliferation in tumors or cells in vitro and in vivo (6-9). The antitumor effect of $ß$-elemene has been demonstrated in the treatment of various tumors, such as glioma, liver cancer, breast carcinoma, leukemia, laryngeal cancer and ovarian cancer (7,10-12). However, the antitumor mechanism of Belemene remains unclear.

Cellular behavior in response to extracellular stimuli can be regulated by the mitogen-activated protein kinase (MAPK) pathways, which participate in the cascade reaction of MAPKKK-MAPKK-MAPK. p38 MAPK, extracellular signalregulated kinase-1 and -2 (ERK1/2) and JNK are at the center of three major MAPK pathways (13-17). Mitogen-activated protein kinase kinase-3 (MKK3) and -6 (MKK6) are two upstream kinases of p38 MAPK and play important roles in regulating cell proliferation, differentiation and apoptosis through phosphorylating the conserved Thr-Gly-Tyr motif in the activation loop of p38 $(18,19)$. MKK3/6 used to be considered two specific activators of p38 MAPK, until recent reports that p38 MAPK also could be activated independently of MKK3/6 through mechanisms beyond the MKKK-MKKMAPK cascade $(20,21)$. In a previous study, we showed that p38 MAPK mediated the anti-glioblastoma effect of ß-elemene (10). Therefore, it was essential to illustrate the role of MKK3/6 in the anti-glioblastoma of $\beta$-elemene. 
In this study, we illustrated the action of MKK3/6 in the anti-glioblastoma proliferation effect of $\beta$-elemene. We found that $B$-elemene inhibited the proliferation of U87 glioblastoma cells and arrested them in G0/G1 phase through up-regulating the expression of phosphorylated MKK3 (p-MKK3) and phosphorylated MKK6 (p-MKK6). In contrast, inhibition of MKK3 and MKK6 with dominant-negative plasmids reversed the antitumor effect of B-elemene. Furthermore, when either MKK3 or MKK6 was inhibited, the other was compensatorily activated in the presence of $ß$-elemene. These results suggest that mutually compensatory activation of MKK3 and MKK6 mediates the anti-glioblastoma of B-elemene. MKK3 and MKK6 might be two putative targets for molecular therapy against glioblastoma.

\section{Materials and methods}

Reagents, antibodies and cell culture. B-Elemene (98\% purity) was purchased from Jingang Pharmaceutical Co. (China). The antibodies against p-MKK3, MKK3, p-MKK6, MKK6 and GAPDH were from Abcam Inc. (UK). Reverse transcription polymerase chain reaction (RT-PCR) kit and primers were from Takara Co., Ltd. (China). Lipofectamine 2000 Transfection Reagent was from Invitrogen Corp. (USA). All other reagents, such as propidium iodide (PI) and RNase, were from Sigma-Aldrich (USA). The human U87 glioblastoma cell line and the rat C6 glioblastoma cell line were purchased from Shanghai Cell Bank of Chinese Academy of Sciences, maintained in Dulbecco's Modified Eagle's Medium supplemented with $10 \%$ fetal calf serum, $50 \mathrm{IU} / \mathrm{ml}$ penicillin and $50 \mathrm{mg} / \mathrm{ml}$ streptomycin, and grown at $37^{\circ} \mathrm{C}$ in a humidified atmosphere with $5 \% \mathrm{CO}_{2}$.

Cell proliferation assay. Cell viability was detected by methyl thiazolyl tetrazolium (MTT) assay. After cells were cultured in the 96-well plate and treated according to needs of the experiment, $0.5 \mathrm{mg} / \mathrm{ml}$ MTT (Sigma-Aldrich, USA) was added to each well, and then the mixture was incubated at $37^{\circ} \mathrm{C}$ for $4 \mathrm{~h}$. Culture medium was replaced with $200 \mu \mathrm{l}$ of dimethyl sulfoxide to dissolve formazan crystals. After the 96-well plate was shaken at room temperature for $10 \mathrm{~min}$, absorbance of each well was determined at $550 \mathrm{~nm}$ using a microplate reader (Bio-Tek, USA). Five replicate wells were designed for each cell sample.

Western blot assay. Cells were extracted with RIPA buffer [50 mM Tris- $\mathrm{HCl}$ (pH 7.4), 0.25\% Na-deoxycholate, $1.0 \%$ NP-40, $1 \mathrm{mM}$ EDTA, $150 \mathrm{mM} \mathrm{NaCl}, 1 \mathrm{mM}$ aprotinin, $1 \mathrm{mg} / \mathrm{ml}$ PMSF, $1 \mu \mathrm{g} / \mathrm{ml}$ leupeptin and $1 \mu \mathrm{g} / \mathrm{ml}$ pepstatin]. The protein concentration in the extracts was determined using the BCA Assay kit (Keygen Biotech. Co., Ltd., China). The proteins were separated in $12 \%$ sodium dodecyl sulfate polyacrylamide gels and transferred to nitrocellulose filter membranes (Bio-Rad, USA). The membranes were blocked with 5\% BSA at room temperature for $2 \mathrm{~h}$ and probed with antibodies to MKK3, p-MKK3, MKK6, p-MKK6 and GAPDH at $4^{\circ} \mathrm{C}$ overnight, then incubated with horseradish peroxidaseconjugated secondary antibodies at $37^{\circ} \mathrm{C}$ for $2 \mathrm{~h}$. Immunoreactive proteins were detected with the ECL system (Amersham, Sweden) and scanned by Image Quant 5.2 software (Amersham).
RNA extraction and RT-PCR. Total RNA was extracted from glioblastoma cells by the routine RNA isolation method using TRIzol (Invitrogen, USA). The quality of RNA samples was qualified using the DU 640 Nucleic Acid and Protein Analyzer (Beckman Coulter, USA). The specific primers for human MKK3, MKK6 and GAPDH were designed by Primer 5.0 software and synthesized by Takara. The sequences of primers were as follows: MKK3: forward 5'-GGTTCCCTTA CGAGTCCTG-3', reverse 5'-TGTCCGTCTTCTTGGTTTT-3'. MKK6: forward 5'-GCAAGAAGCGAAACCCTG-3', reverse 5'-CTGGGAGTTGTGGAGACG-3'. GAPDH: forward 5'-AAC GGATTTGGTCGTATTG-3', reverse 5'-GCTCCTGGAAG ATGGTGAT-3'. RT-PCR was carried out with an RT-PCR kit (Takara) according to the manufacturer's instructions.

Transfection. For transient transfection, cells were plated in 6-well plates at a density of $4 \times 10^{5}$ and cultured for $24 \mathrm{~h}$. Empty vector, dominant-negative MKK3 (DN-MKK3) and dominant-negative MKK6 (DN-MKK6) plasmids (marked with an HA tag) were transfected into glioblastoma cells with Lipofectamine 2000 according to the manufacturer's instructions. The DN-MKK3 and DN-MKK6 plasmids were generously provided by Dr Jim Woodgett (Ontario Cancer Institute, Princess Margaret Hospital, Canada).

Immunofluorescence and 4'-6-diamidino-2-phenylindole (DAPI) staining. In each well of 24 -well plates, $2 \times 10^{5}$ U87 cells were plated with polylysine-coated cover slips and cultured for $24 \mathrm{~h}$. Cells were transfected with Lipofectamine 2000 for 24 h. Cells on cover slips were washed with PBS, fixed with methanol and then treated with $0.1 \%$ Triton X-100. After blocking with 5\% BSA, cells were incubated with anti-HA tag antibody at a 1:200 dilution (Millipore, USA) at $4^{\circ} \mathrm{C}$ overnight and labeled with FITC-conjugated antibody to mouse $\mathrm{IgG}$ for $40 \mathrm{~min}$ at $37^{\circ} \mathrm{C}$ in the dark. Cell nuclei were stained with DAPI (Sigma-Aldrich, USA), and immunofluorescent images were examined using a fluorescent microscope (Leica Microsystems, Germany).

Cell cycle analysis. U87 cells were plated at a density of $4 \times 10^{5}$ in 6-well plates. When cells were 90-95\% confluent, transfection was carried out for $24 \mathrm{~h}$ with Lipofectamine 2000. After treatment with $60 \mu \mathrm{g} / \mathrm{ml}$ B-elemene for $24 \mathrm{~h}$, cells were harvested and washed with PBS and then fixed with $70 \%$ ethanol at $-20^{\circ} \mathrm{C}$ overnight. U87 cells were stained with PI after incubating in $0.2 \mathrm{mg} / \mathrm{ml} \mathrm{RNase}$ at $37^{\circ} \mathrm{C}$ for $1 \mathrm{~h}$. Finally, cell samples were analyzed by a FACSCalibur (BectonDickinson, USA). Cell cycle phase distributions were analyzed with CellQuest Pro software (Becton-Dickinson) and ModFit LT software (Verity Software House, USA).

Patients and specimens. Tumor tissues originated from 20 patients ( 9 men and 11 women, $47 \pm 12.7$ years) with glioblastoma who had undergone tumorectomy. Normal brain tissues were from 19 subjects ( 9 men and 10 women, $44 \pm 13.1$ years) undergoing intracranial decompression without brain tumors between 2008 and 2010 at the First Affiliated Hospital of Dalian Medical University. The study was approved by the Ethics Committee of Dalian Medical University and abided by the Declaration of Helsinki, with informed consent obtained from all study participants. 
A

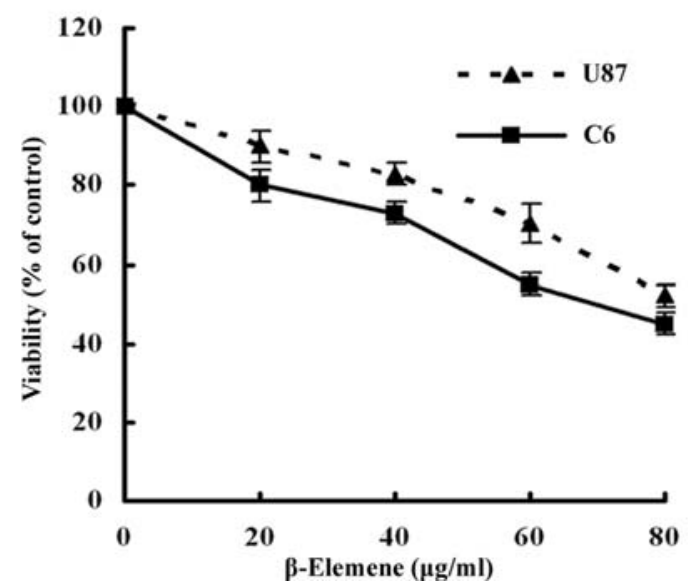

B

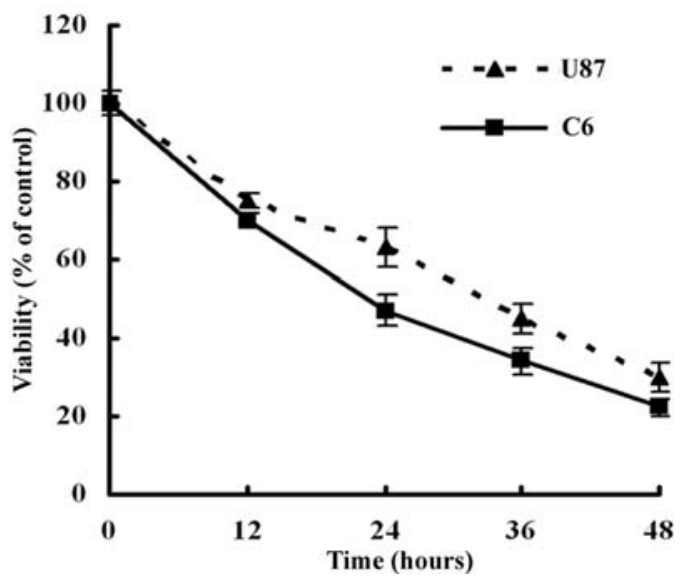

Figure 1. B-Elemene inhibits C6 and U87 cell proliferation. (A) C6 and U87 cells were treated with B-elemene at different concentrations (0, $20,40,60$ and $80 \mu \mathrm{g} / \mathrm{ml}$ ) for $24 \mathrm{~h}$, and then cell viability was determined by MTT assay. (B) C6 and U87 cells were treated by 3 -elemene for different times $(0,12,24,36$ and $48 \mathrm{~h}$ ) at a concentration of $60 \mu \mathrm{g} / \mathrm{ml}$, and then cell viability was determined by MTT assay. The viability of $\beta$-elemene-treated cells decreased with increasing drug dose or treatment time.

Immunohistochemical analysis. Paraffin sections of normal brain and glioblastoma tissues were obtained and deparaffinized. Antigen retrieval was performed with citrate buffer $\mathrm{pH} 6.0$ (Invitrogen). Non-specific sites were blocked by incubating sections with 5\% BSA in a humidified chamber for $1 \mathrm{~h}$ at room temperature. The samples were incubated with $0.3 \%$ $\mathrm{H}_{2} \mathrm{O}_{2}$ for 15 min to block endogenous peroxidase activity and then labeled with different primary antibodies at proper concentrations (1:50 for p-MKK3, p-MKK6, MKK6 and 1:25 for MKK3) for $1 \mathrm{~h}$ at room temperature. In subsequent steps, we used the Vectastain ABC kit (Vector Laboratories, USA) and DAB as a chromogen (Changdao Biotech, China). Nuclear counterstaining of sections was performed with hematoxylin. In each tissue, additional staining without primary antibody was carried out in parallel as a negative control. The integrated optical density (IOD) was used to semi-quantitatively estimate the expression of $\mathrm{p}-\mathrm{MKK} 3 / 6$ and MKK3/6 in Image-Pro Plus 6.0 software.

Statistical analysis. All values were expressed as means \pm SD. Statistical analysis was performed by Student's t-test. Statistical significance was defined as $\mathrm{p}<0.05$ between different groups, and $\mathrm{p}<0.01$ was highly significant. Statistical analysis was done with SPSS software (SPSS, Inc., USA).

\section{Results}

$\beta$-Elemene inhibits growth of both human U87 and rat C6 glioblastoma cells. To evaluate the anti-glioblastoma effect of ß-elemene, rat C6 and human U87 glioblastoma cells were treated with $\beta$-elemene at different doses or for different durations, and then a MTT assay was performed to determine cell viability. We found that viability of $\beta$-elemene-treated cells decreased with increasing drug dose (Fig. 1A) or treatment time (Fig. 1B). These results indicate that proliferation of glioblastoma cells was inhibited dose- and time-dependently by ß-elemene.
$\beta$-Elemene increases phosphorylation of both MKK3 and MKK6 in human glioblastoma cells. We reported previously that p38 MAPK played an essential part in inhibiting glioblastoma cellular proliferation and arresting cell cycle progression in the G0/G1 phase by B-elemene (10). MKK3 and MKK6 usually are considered specific upstream kinases of p38 MAPK. However, recent research has shown that they are not essential for activation of p38 MAPK $(20,21)$, so it is not certain whether MMK3/6 mediates the involvement of the MAPK pathway in the anti-glioblastoma effect of $\beta$-elemene. We thus examined activity of MKK3/6 in U87 glioblastoma cells treated with different concentrations of B-elemene for $1 \mathrm{~h}$ or $24 \mathrm{~h}$ by Western blot or RT-PCR assay, respectively. As shown in Fig. 2A, phosphorylated levels of both MKK3 and MKK6 were increased in a dose-dependent manner. However, the expression of total MKK3/6 was not affected by $\beta$-elemene treatment for either $1 \mathrm{~h}$ (Fig. 2A) or $24 \mathrm{~h}$ (Fig. 2B). The Western blot analysis results were further semi-quantitatively estimated using Gel-Pro Analyzer 4.0 software, and the changes in the levels of p-MKK3, MKK3, p-MKK6 and MKK6 were illustrated by a polygram (Fig. 2C). These results suggest that $\beta$-elemene increased the activity of MKK3/6 to inhibit proliferation of U87 cells.

Inhibition of MKK3 and MKK6 blocks the anti-glioblastoma proliferation effect of $\beta$-elemene in vitro. To test whether MKK3/6 is critical for the $\beta$-elemene-induced anti-proliferative effect, we transfected U87 glioblastoma cells with DN-MKK3, DN-MKK6 and empty plasmids, and then examined transfection efficiency by fluorescence microscopy and cell growth by MTT assay. As shown in our results, mutation plasmids were transfected efficiently into U87 cells (Fig. 3A). The cell proliferation activities in all DN-MKK3 (Fig. 3B, bar c), DNMKK6 (Fig. 3B, bar d) and DN-MKK3 + DN-MKK6 (Fig. 3B, bar e) cells were higher than those transfected with empty vector (Fig. 3B, bar b). These results suggest that the antiproliferation effect of $\beta$-elemene was distinctly inhibited by 
$\mathbf{A}$

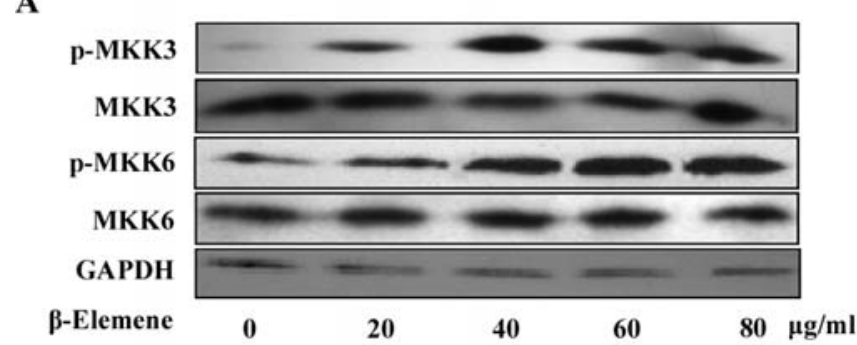

B

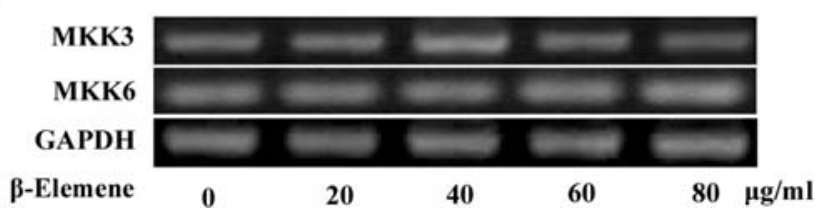

C

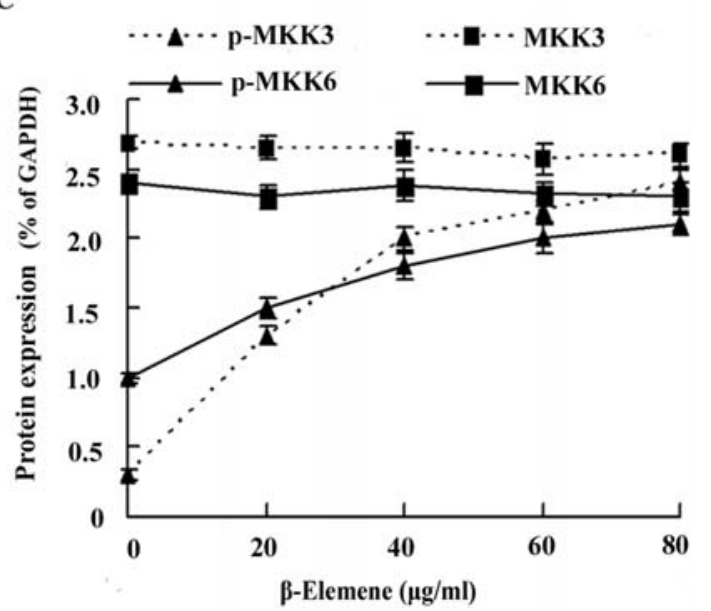

Figure 2. B-Elemene increased phosphorylation of MKK3 and MKK6. (A) U87 cells were treated with different concentrations of $\beta$-elemene for $1 \mathrm{~h}$ and extracted with protein lysis buffer. p-MKK3, MKK3, p-MKK6 and MKK6 were measured by Western blot analysis. p-MKK3 and p-MKK6 (and not total MKK3 or MKK6) were enhanced dose-dependently by $\beta$-elemene. (B) After U87 cells were treated with various concentrations of $\beta$-elemene for $24 \mathrm{~h}$, total RNA was extracted. RT-PCR was performed using specific primers for MKK3, MKK6 and GAPDH. Expression of total MKK3 and MKK6 was not affected by 24-h B-elemene. (C) The Western blot results (A) were further analyzed using Gel-Pro Analyzer 4.0 software. The changes in the levels of p-MKK3, MKK3, p-MKK6 and MKK6 were illustrated by a polygram. The results are representative of three independent experiments.

A

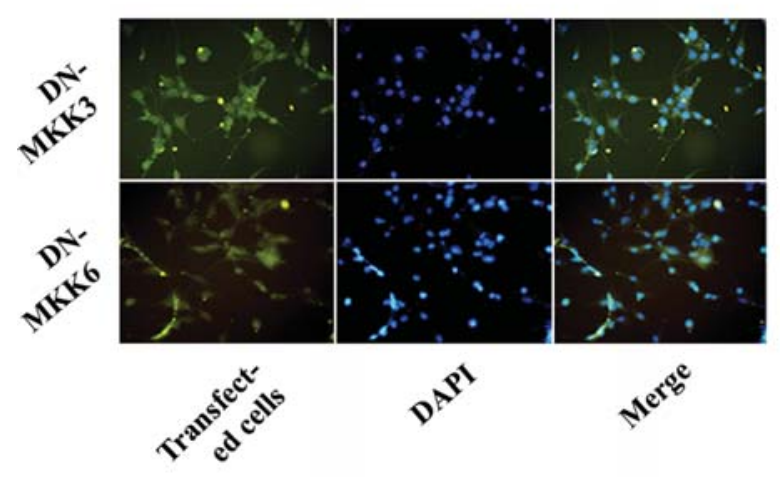

B

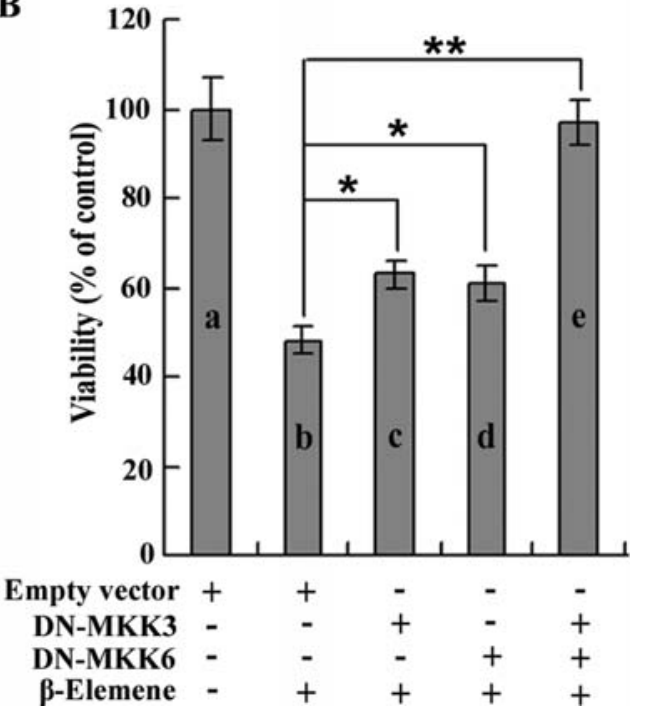

Figure 3. Transfection with DN-MKK3 and DN-MKK6 plasmids inhibited the B-elemene-induced anti-proliferation effect in U87 cells. (A) After transfection with DN-MKK3 or DN-MKK6, U87 cells were labeled with anti-HA tag antibody and stained with DAPI. A high transfection efficiency was confirmed using a fluorescence microscope. (B) Empty vector, DN-MKK3, DN-MKK6 or DN-MKK3 + DN-MKK6 were transfected into U87 cells prior to $24-\mathrm{h}$ treatment with $60 \mu \mathrm{g} / \mathrm{ml}$ B-elemene. Proliferation activity was measured by MTT assay. Although DN-MKK3 (B, bar c) or DN-MKK6 (B, bar d) alone partially inhibited the action of $\beta$-elemene $(\mathrm{p}<0.05)$, co-transfection $(\mathrm{B}$, bar e) with these two mutant plasmids nearly completely blocked the anti-proliferation effect of $\beta$-elemene $(\mathrm{p}<0.01)$. Data shown represent means $\pm \mathrm{SD}$ from three independent experiments performed in triplicate $\left({ }^{*} \mathrm{p}<0.05,{ }^{* *} \mathrm{p}<0.01\right)$.

blocking the MKK3/6 pathway. Therefore, the inhibitory effect of B-elemene on the growth of human glioblastoma cells depended on activation of the MKK3/6 pathway.

\section{Inhibition of MKK3 and MKK6 blocked $\beta$-elemene-induced} G0/G1-phase arrest in human glioblastoma cells. To explore the mechanism by which $\beta$-elemene inhibits glioblastoma cell growth, we evaluated cell cycle progression by flow cytometry after U87 cells were transfected with five combinations of plasmids and treated with $60 \mu \mathrm{g} / \mathrm{ml} \mathrm{B-elemene} \mathrm{for} 24 \mathrm{~h}$. The percentages of the five groups of cells in G0/G1 phase were illustrated in Fig. 4F. The fraction of U87 cells in the G0/G1 phase was higher in the B-elemene-treated group (Fig. 4A) than the B-elemene-untreated group (Fig. 4E, $\mathrm{p}<0.01)$. The percentage of U87 cells in $\mathrm{G} 0 / \mathrm{G} 1$ phase in all the DN-MKK3 (Fig. 4C), DN-MKK6 (Fig. 4B) and DN-MKK3/6 co-transfected cells (Fig. 4D) was lower than in the empty vector group treated by $\beta$-elemene (Fig. 4A). These results 
A

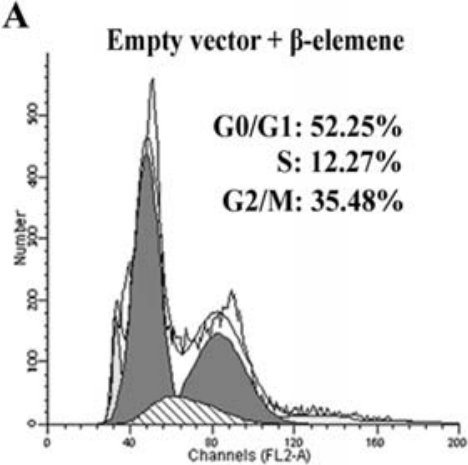

D

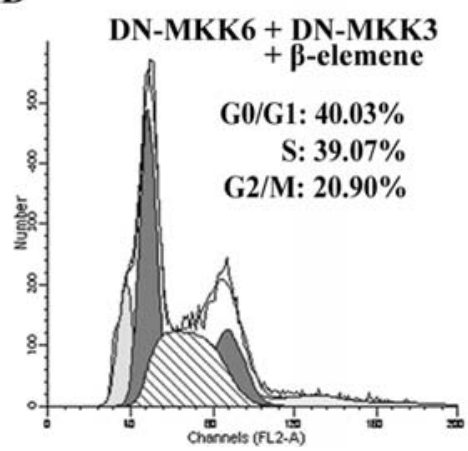

B

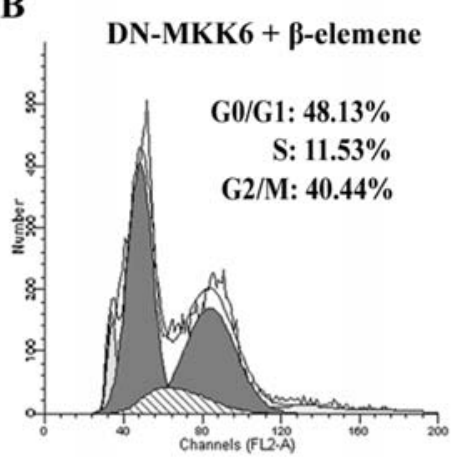

$\mathbf{E}$

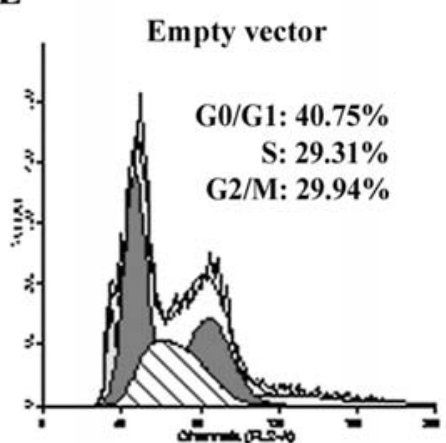

C
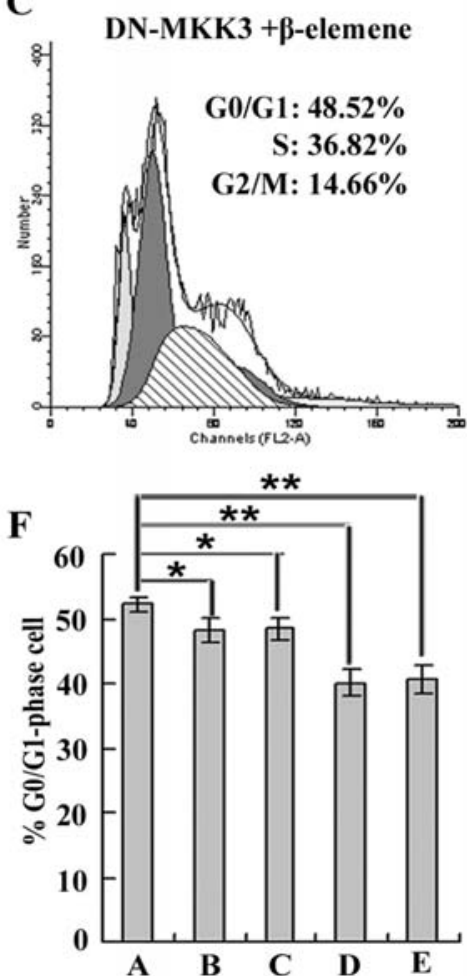

Figure 4. Transfection with DN-MKK3 and DN-MKK6 plasmids reverses ß-elemene-induced G0/G1-phase arrest in U87 cells. (A-E) After transfection with different plasmids for $24 \mathrm{~h}$, U87 cells were treated with $60 \mu \mathrm{g} / \mathrm{ml}$ ß-elemene for $24 \mathrm{~h}$, and then cell cycle progression was determined by flow cytometry. (F) The percentages of all groups of cells in G0/G1 phase were illustrated by a vertical bar chart. The fraction of U87 cells in G0/G1 phase was higher in the B-elemene-treated group (A) than the B-elemene-untreated group (E, p<0.01). The percentages of U87 cells in G0/G1 phase in the DN-MKK3 (C), DN-MKK6 (B) and co-transfected group (D) were lower than in the empty vector group treated by $\beta$-elemene (A). The data are representative of three independent experiments $\left({ }^{*} \mathrm{p}<0.05,{ }^{* *} \mathrm{p}<0.01\right)$.

A

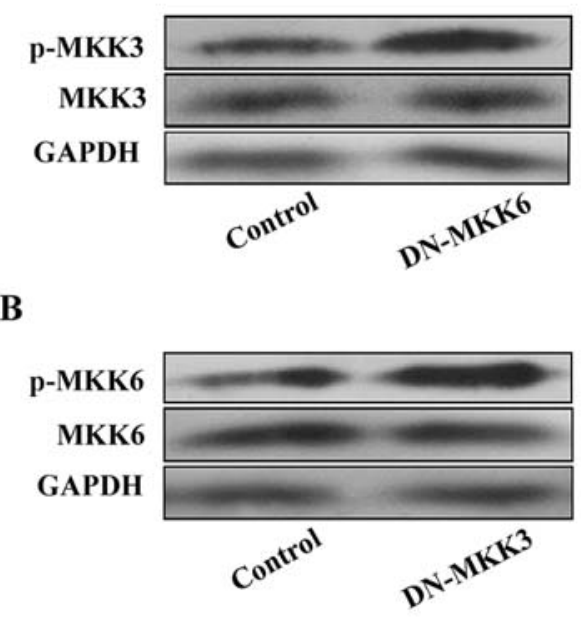

C

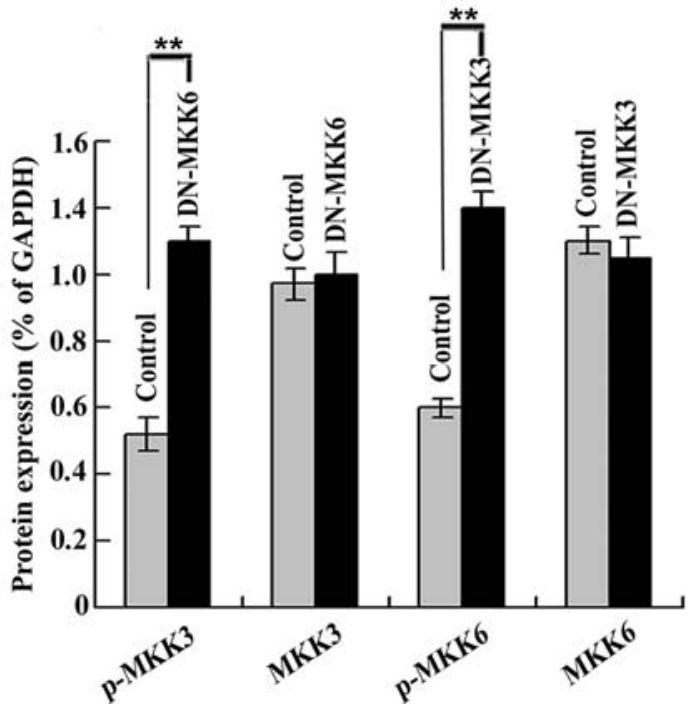

Figure 5. When either MKK3 or MKK6 was inhibited by a dominant-negative construct, the activity of the other was increased compensatorily by $\beta$-elemene. U87 cells were transfected with DN-MKK3 or DN-MKK6 for $24 \mathrm{~h}$ (control, empty vector) and treated with $60 \mu \mathrm{g} / \mathrm{ml}$ ß-elemene for $24 \mathrm{~h}$. p-MKK6/3 and total MKK6/3 proteins were measured by Western blot assay. (A) Under the action of ß-elemene, p-MKK3 compensatorily increased when MKK6 was inhibited by DN-MKK6. (B) Under the action of $\beta$-elemene, p-MKK6 compensatorily increased when MKK3 was inhibited by DN-MKK3. (C) The results of Western blots were analyzed with Gel-Pro Analyzer 4.0 software. The alterations in protein level were illustrated by a vertical bar chart. The results are representative of three independent experiments $\left({ }^{* *} \mathrm{p}<0.01\right)$.

suggest that $\beta$-elemene-induced inhibition of U87 cell proliferation was likely due to G0/G1-phase arrest mediated by activation of MKK3/6 pathway.
Compensatory increases in MKK3 or MKK6 phosphorylation by $\beta$-elemene when the other was inhibited with a dominantnegative plasmid. We were intrigued by the findings from the 
A
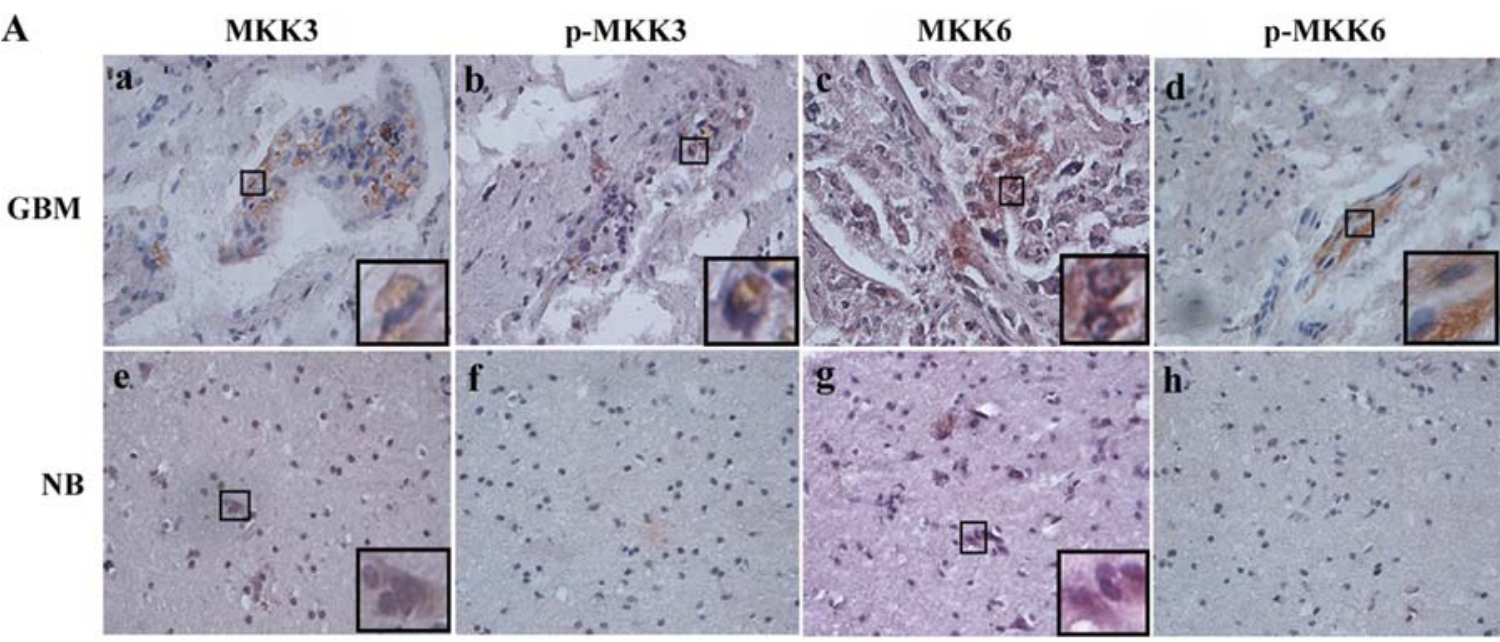

B

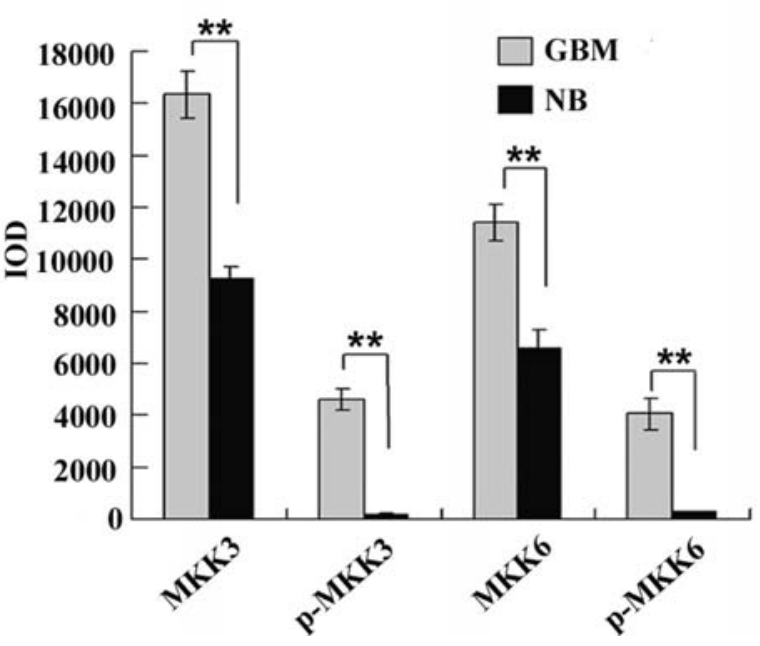

Figure 6. Immunohistochemical analysis of glioblastoma (GBM) and normal brain (NB) tissue to determined the expression of MKK3/6 and p-MKK3/6. (A) After tissues were prepared following routine protocols, sample slides were detected using antibodies of MKK3, p-MKK3, MKK6 and p-MKK6 by immunohistochemistry. IOD was analyzed using Image-Pro Plus 6.0 software (the same selected areas and magnifications in all the images). Individual tumor cells (inset black squares) were magnified to facilitate interpretation of the staining pattern (A, panels a-e and g). (B) Each group IOD was illustrated by a vertical bar chart. IOD for MKK3, p-MKK3, MKK6 and p-MKK6 were higher significantly in GBM groups (A, panels a-d) than NB groups (A, panels e-h) $\left({ }^{* *} \mathrm{p}<0.01\right)$. Serial sections stained with control antibodies were negative (data not shown). Original magnifications, $\mathrm{x} 200$.

cell viability and cell cycle arrest assays that co-transfecting DN-MKK3 and DN-MKK6 offset the effect of B-elemene on U87 cells more markedly than expected from the additive effect of the DN-MKK3 and DN-MKK6 single transfections. Thus, we supposed that some redundancy might exist between MKK3 and MKK6. To elucidate this relationship, U87 cells were transfected with DN-MKK3 or DN-MKK6 for $24 \mathrm{~h}$ (with the empty vector as control) and then treated with $60 \mu \mathrm{g} / \mathrm{ml} \mathrm{B-elemene} \mathrm{for} 24 \mathrm{~h}$. p-MKK6/3 and total MKK6/3 proteins were measured in the lysis products by Western blot analysis (Fig. 5A and B). The results of the Western blots were analyzed using Gel-Pro Analyzer 4.0 software. The alterations in protein levels were illustrated by a vertical bar chart (Fig. 5C). We found that under the action of $\beta$-elemene, the expression of p-MKK3 was higher in DN-MKK6 cells than controls $(\mathrm{p}<0.01)$, and p-MKK6 was also significantly increased in DN-MKK3 cells in comparison with controls $(\mathrm{p}<0.01)$. Therefore, when either MKK3 or MKK6 was inhibited by a dominant-negative construct in U87 cells, the other was activated compensatorily by $ß$-elemene.

Overexpression of $M K K 3 / 6$ and $p-M K K 3 / 6$ in human glioblastoma tissue. To evaluate the expression levels of MKK3/6 and p-MKK3/6 in human glioblastoma tissue, immunohistochemistry on glioblastoma and normal brain tissues was performed using antibodies against MKK3, p-MKK3, MKK6 and p-MKK6 (Fig. 6A). Image-Pro Plus 6.0 software was applied to calculate the IOD of each group (the same selected areas and magnifications in all images), and then statistical analysis was performed. Each group's IOD was illustrated in a vertical bar chart (Fig. 6B). We found in this assay that the IOD values reflecting the expression of MKK3/6 and pMKK3/6 in the glioblastoma (abbreviated 'GBM' in Fig. 6A, panels a-d) group were significantly higher than in the normal brain group (abbreviated 'NB' in Fig. 6A, panels e-h) $(p<0.01)$. These results suggest that the expression of both 
total and phosphorylated forms of MKK3/6 were up-regulated in human glioblastoma.

\section{Discussion}

p38 MAPK, ERK and JNK are three major MAPKs that regulate mammalian cellular behaviors. As two important members of the MKKK-MKK-MAPK cascade reaction, MKK3 and MKK6 can activate the p38 MAPK pathway to regulate cellular growth, fission, differentiation and apoptosis. MKK3/6 can be activated through phosphorylation by MKKKs. Those MKKKs responsible for activating the MKK3/6-p38 MAPK pathways seem to be cell type-specific and stimulus-specific, including TAK1, ASK1, MLKs and some members of the MEKK family $(22,23)$. Previously, we reported that $\mathrm{p} 38$ MAPK mediated the anti-glioblastoma action of $\beta$-elemene. Although MKK3/6 used to be considered two specific kinases of p38, p38 is now known to be also activated through MKK3/6-independent mechanisms $(20,21)$. Therefore, MKK3/6 is the key factor in our research of the anti-glioblastoma mechanisms of $B$-elemene. The present study shows that $\beta$-elemene inhibited proliferation of glioblastoma cells and arrested cells in G0/G1 through activating the MKK3/6 pathway. In contrast, inhibition of MKK3 and MKK6 reversed the anti-glioblastoma effect of B-elemene. These results indicate that the anti-glioblastoma action of Belemene depends on activation of MKK $3 / 6$ in vitro. This conclusion is supported by the fact that MKK3/6 also frequently mediates the antitumor effects of various drugs, such as isoflavone derivative, sodium butyrate, TNF- $\alpha$ and gemcitabine (24-27). Therefore, considering the key role in inhibiting the proliferation of human glioblastoma cells, activation of MKK3/6 by B-elemene provides an important strategy for molecular therapy against glioblastoma.

Many studies have shown that the activities of p38 MAPK, ERK and JNK are altered in various malignant tumors, such as breast carcinoma, brain cancer, hepatoma and renal carcinoma (28-35). Altered activity of MKK3/6 has also been seen in carcinomas of breast, brain, prostate, liver and other tissues (28-31). To further illustrate the relationship between MKK3/6 and glioblastoma, MKK3/6 and p-MKK3/6 in human glioblastoma tissues were determined by immunohistochemistry. The expression of both MKK3/6 and p-MKK3/6 were increased in human glioblastoma tissues, suggesting that the MKK3/6 pathway might be related to the development of human glioblastoma. However, it is worth noting that the roles of MKK3/6 activation in glioblastoma development and in mediating the anti-glioblastoma effect of $\beta$-elemene are not necessarily functionally contradictory, as a similar dichotomy has been seen with ERK in breast cancer $(28,36)$ and p38 MAPK in glioma $(29,10)$. The defensive activation of MKK3/6 or other more complicated mechanisms cannot be ruled out in the development of glioblastoma. In short, although more clinical samples and animal experiments are still needed, our results enhance the rationality of molecular therapies for glioblastoma that target MKK3/6.

In addition, the data presented here show that combined inhibition of MKK3 and MKK6 counteracted the anti-glioblastoma effect of ß-elemene more effectively than blocking MKK3 or MKK6 singly, and it even surpassed the additive effect of
MKK3 and MKK6 inhibition. Our further investigations indicated that MKK3 and MKK6 compensated functionally for each other when one of them was inhibited. Although the compensatory mechanism between MKK3 and MKK6 is still unclear, negative feedback in the Ras/MKK3/ p38-JNKMKK1/2/ERK pathway has been reported (37), which partially illuminates the potentially complicated cross-talk among MKK/MAPK pathways. In the future, the compensatory mechanism or negative feedback in which MKK $3 / 6$ participates should be worth studying in molecular therapies targeting MKK3/6.

In conclusion, we propose that mutually compensatory activation of MKK3 and MKK6 mediates the antiglioblastoma effect of $\beta$-elemene. MKK3 and MKK6 are involved in the development of human glioblastoma, and might be two novel molecular targets for glioblastoma therapy.

\section{Acknowledgements}

This research was supported by the National Natural Science Foundation of China (no. 30740027/30471778). We also thank all our colleagues in our research group for their generous support.

\section{References}

1. Louis DN, Ohgaki H, Wiestler OD, Cavenee WK, Burger PC, Jouvet A, Scheithauer BW and Kleihues P: The 2007 WHO classification of tumours of the central nervous system. Acta Neuropathol 114: 97-109, 2007.

2. Wen PY and Kesari S: Malignant gliomas in adults. N Engl J Med 359: 492-507, 2008.

3. Kang TY, Jin T, Elinzano H and Peereboom D: Irinotecan and bevacizumab in progressive primary brain tumors, an evaluation of efficacy and safety. J Neurooncol 89: 113-118, 2008.

4. Clarke J, Butowski N and Chang S: Recent advances in therapy for glioblastoma. Arch Neurol 67: 279-283, 2010.

5. Chen SL, You J and Wang GJ: Supercritical fluid extraction of B-elemene under lower pressure. Se Pu 19: 179-181, 2001.

6. Wang G, Li X, Huang F, Zhao J, Ding H, Cunningham C, Coad JE, Flynn DC, Reed E and Li QQ: Antitumor effect of Belemene in non-small-cell lung cancer cells is mediated via induction of cell cycle arrest and apoptotic cell death. Cell Mol Life Sci 62: 881-893, 2005.

7. Li X, Wang G, Zhao J, Ding H, Cunningham C, Chen F, Flynn DC, Reed E and Li QQ: Antiproliferative effect of Belemene in chemoresistant ovarian carcinoma cells is mediated through arrest of the cell cycle at the G2-M phase. Cell Mol Life Sci 62: 894-904, 2005.

8. Xu L, Tao S, Wang X, Yu Z, Wang M, Chen D, Jing Y and Dong J: The synthesis and anti-proliferative effects of $\beta$ elemene derivatives with mTOR inhibition activity. Bioorg Med Chem 14: 5351-5356, 2006.

9. Zhao J, Li QQ, Zou B, Wang G, Li X, Kim JE, Cuff CF, Huang L, Reed $\mathrm{E}$ and Gardner K: In vitro combination characterization of the new anticancer plant drug beta-elemene with taxanes against human lung carcinoma. Int J Oncol 31: 241-252, 2007.

10. Yao YQ, Ding X, Jia YC, Huang CX, Wang YZ and Xu YH: Anti-tumor effect of $\beta$-elemene in glioblastoma cells depends on p38 MAPK activation. Cancer Lett 264: 127-134, 2008.

11. Peng X, Zhao Y, Liang X, Wu L, Cui S, Guo A and Wang W: Assessing the quality of RCTs on the effect of B-elemene, one ingredient of a Chinese herb, against malignant tumors. Contemp Clin Trials 27: 70-82, 2006.

12. Tao L, Zhou L, Zheng L and Yao M: Elemene displays anticancer ability on laryngeal cancer cells in vitro and in vivo. Cancer Chemother Pharmacol 58: 24-34, 2006.

13. Suzuki A, Guicheux J, Palmer G, Miura Y, Oiso Y, Bonjour JP and Caverzasio J: Evidence for a role of p38 MAP kinase in expression of alkaline phosphatase during osteoblastic cell differentiation. Bone 30: 91-98, 2002. 
14. Molton SA, Todd DE and Cook SJ: Selective activation of the c-Jun N-terminal kinase (JNK) pathway fails to elicit Bax activation or apoptosis unless the phosphoinositide 3'-kinase (PI3K) pathway is inhibited. Oncogene 22: 4690-4701, 2003.

15. Hohmann S: Osmotic adaptation in yeast-control of the yeast osmolyte system. Int Rev Cytol 215: 149-187, 2002.

16. Davis RJ: Signal transduction by the JNK group of MAP kinases. Cell 103: 239-252, 2000.

17. Chang L and Karin M: Mammalian MAP kinase signalling cascades. Nature 410: 37-40, 2001.

18. Kang YJ, Seit-Nebi A, Davis RJ and Han J: Multiple activation mechanisms of $\mathrm{p} 38$ ? mitogen-activated protein kinase. J Biol Chem 281: 26225-26234, 2006.

19. Dérijard B, Raingeaud J, Barrett T, Wu IH, Han J, Ulevitch RJ and Davis RJ: Independent human MAP-kinase signal transduction pathways defined by MEK and MKK isoforms. Science 267: 682-685, 1995

20. Ge B, Gram H, Di Padova F, Huang B, New L, Ulevitch RJ, Luo Y and Han J: MAPKK-independent activation of p38 $\alpha$ mediated by TAB1-dependent autophosphorylation of $\mathrm{p} 38 \alpha$. Science 295: 1291-1294, 2002.

21. Gills JJ, Castillo SS, Zhang C, Petukhov PA, Memmott RM, Hollingshead M, Warfel N, Han J, Kozikowski AP and Dennis PA: Phosphatidylinositol ether lipid analogues that inhibit AKT also independently activate the stress kinase, $\mathrm{p} 38 \alpha$, through MKK3/6-independent and -dependent mechanisms. J Biol Chem 282: 27020-27029, 2007.

22. Kyriakis JM and Avruch J: Mammalian mitogen-activated protein kinase signal transduction pathways activated by stress and inflammation. Physiol Rev 81: 807-869, 2001.

23. Cheung PC, Campbell DG, Nebreda AR and Cohen P: Feedback control of the protein kinase TAK1 by SAPK2a/p38 . EMBO J 22: 5793-5805, 2003.

24. Chen JT, Fong YC, Li TM, Liu JF, Hsu CW, Chang CS and Tang CH: DDTD, an isoflavone derivative, induces cell apoptosis through the reactive oxygen species/apoptosis signal-regulating kinase 1 pathway in human osteosarcoma cells. Eur J Pharmacol 597: 19-26, 2008.

25. Cho SD, Ahn NS, Jung JW, Yang SR, Park JS, Lee YS, Jo EH, Hwang JW, Lii J and Kang KS: Critical role of the c-JunNH2terminal kinase and p38 mitogen-activated protein kinase pathways on sodium butyrate-induced apoptosis in DU145 human prostate cancer cells. Eur J Cancer Prev 15: 57-63, 2006.

26. Liu AH, Deng P and Jiang Y: The TNF- $\alpha$-induced apoptosis of LA795 cells is mediated by MAP kinase kinase 6 . Zhonghua Jie He He Hu Xi Za Zhi 26: 88-92, 2003.

27. Habiro A, Tanno S, Koizumi K, Izawa T, Nakano Y, Osanai M, Mizukami Y, Okumura T and Kohgo Y: Involvement of p38 mitogen-activated protein kinase in gemcitabine-induced apoptosis in human pancreatic cancer cells. Biochem Biophys Res Commun 316: 71-77, 2004.
28. Wang HY, Cheng Z and Malbon CC: Overexpression of mitogenactivated protein kinase phosphatases MKP1, MKP2 in human breast cancer. Cancer Lett 191: 229-237, 2003.

29. Demuth T, Reavie LB, Rennert JL, Nakada M, Nakada S, Hoelzinger DB, Beaudry CE, Henrichs AN, Anderson EM and Berens ME: MAP-ing glioma invasion: Mitogen-activated protein kinase kinase 3 and p38 drive glioma invasion and progression and predict patient survival. Mol Cancer Ther 6: 1212-1222, 2007.

30. Lotan TL, Lyon M, Huo D, Taxy JB, Brendler C, Foster BA, Stadler W and Rinker-Schaeffer CW: Up-regulation of MKK4, MKK6 and MKK7 during prostate cancer progression: an important role for SAPK signalling in prostatic neoplasia. J Pathol 212: 386-394, 2007.

31. Iyoda K, Sasaki Y, Horimoto M, Toyama T, Yakushijin T, Sakakibara M, Takehara T, Fujimoto J, Hori M, Wands JR and Hayashi N: Involvement of the p38 mitogen-activated protein kinase cascade in hepatocellular carcinoma. Cancer 97: 3017-3026, 2003.

32. Zhang J, Zhi H, Zhou C, Ding F, Luo A, Zhang X, Sun Y, Wang $\mathrm{X}, \mathrm{Wu} \mathrm{M}$ and Liu Z: Up-regulation of fibronectin in cesophageal squamous cell carcinoma is associated with activation of the Erk pathway. J Pathol 207: 402-409, 2005.

33. Samaras V, Tsopanomichalou M, Stamatelli A, Arnaoutoglou C, Samaras E, Arnaoutoglou M, Poulias H and Barbatis C: Is there any potential link among caspase-8, p-p38 MAPK and bcl-2 in clear cell renal cell carcinomas? A comparative immunohistochemical analysis with clinical connotations. Diagn Pathol 4: 7, 2009.

34. Handra-Luca A, Mauguen A, Ménard P and Fouret P: Coordinated expression of activated mitogen-activated protein kinases in salivary gland adenoid cystic carcinoma. Hum Pathol 39: 1590-1596, 2008

35. Lama G, Mangiola A, Anile C, Sabatino G, De Bonis P, Lauriola L, Giannitelli C, La Torre G, Jhanwar-Uniyal M, Sica G and Maira G: Activated ERK1/2 expression in glioblastoma multiforme and in peritumor tissue. Int J Oncol 30: 1333-1342, 2007.

36. Rojo F, González-Navarrete I, Bragado R, Dalmases A, Menéndez S, Cortes-Sempere M, Suárez C, Oliva C, Servitja S, Rodriguez-Fanjul V, Sánchez-Pérez I, Campas C, Corominas JM, Tusquets I, Bellosillo B, Serrano S, Perona R, Rovira A and Albanell J: Mitogen-activated protein kinase phosphatase-1 in human breast cancer independently predicts prognosis and is repressed by doxorubicin. Clin Cancer Res 15: 3530-3539, 2009.

37. Chen G, Hitomi M, Han J and Stacey DW: The p38 pathway provides negative feedback for Ras proliferative signaling. J Biol Chem 275: 38973-38980, 2000. 